\title{
Clinical Evaluation of Two Prosthetic Suspension Systems in a Bilateral Transtibial Amputee
}

Authors:

Hossein Gholizadeh, MEngSc

Noor Azuan Abu Osman, PhD

Mojtaba Kamyab, PhD

Arezoo Eshraghi, $\mathrm{PhD}$

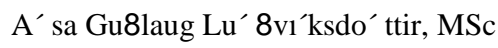

Wan Abu Bakar Wan Abas, PhD

Affiliations:

From the Department of Biomedical

Engineering, Faculty of Engineering,

University of Malaya, Kuala Lumpur,

Malaysia (HG, NAAO, AE, WABWA);

Department of Orthotics and

Prosthetics, Faculty of Rehabilitation

Sciences, Tehran University of Medical

Sciences, Tehran, Iran (MK); and

Clinical Research, R\&D, Ossur Head

Office, Reykjavi 'k, Iceland (A'GL).

Correspondence:

All correspondence and requests for reprints should be addressed to:

Hossein Gholizadeh, MEngSc,

Department of Biomedical

Engineering, Faculty of Engineering,

University of Malaya, Kuala Lumpur,

50603 Malaysia.

\section{Disclosures:}

Supported by Malaysia UM/MOHE/HIR

Project No. D000014-16001 and

O”ssur (Reykjavik, Iceland). Financial

disclosure statements have been 
obtained, and no conflicts of interest

have been reported by the authors or

by any individual in control of the

content of this article.

0894-9115/12/9110-0894/0

American Journal of Physical

Medicine \& Rehabilitation

DOI: 10.1097/PHM.0b013e31823c74d7

rosthetic suspension plays a significant role in ensuring the secure attachment

of prosthesis to the residual limb. There is a strong correlation between

the vertical movement within the socket, or pistoning, and the prosthetic suspension

method. There is also evidence that patient satisfaction is associated

with appropriate suspension.1Y3 Therefore, it is recognized that pistoning measurement

is helpful for clinicians and researchers who wish to improve suspension

systems and decrease the adverse effects of pistoning movement.

Prosthetists rely on their experience and the technical information provided

by manufacturer to choose appropriate liners for their patients.6 There is

a wide variety of suspension systems available for lower limb prostheses, of

which, silicon liners are frequently used.1 Silicon liners were first introduced

in 1986. Their main advantage was claimed to be better suspension compared with other soft sockets such as polyethylene foam (Pelite) liners because of enhanced bond with the

residual limb.7Y10 When attempting to understand

the effectiveness of a prosthetic suspension system, the amount of pistoning may be considered as an indicator.9 One of the most recent prosthetic liner types, the Seal-In X5 liner, is a suction suspension liner that provides a hypobaric sealing membrane around the silicon liner without an external sleeve or shuttle lock (Fig. 1AYD). It was invented by O"ssur (Reykjavik, Iceland) to reduce the pistoning movement inside the socket through increased contact surface with the socket wall. It is also said to distribute pressure evenly in a manner that prevents discomfort at the end of the residual limb.

In the literature review, no comparative study was found regarding the effect of Seal-In X5 and 
locking liners on prosthetic suspension and satisfaction.

Therefore, the purpose of this study was

to clinically investigate the effects of two suspension

systems on a subject with bilateral transtibial

amputation.

\section{CASE REPORT}

This report describes the study that was conducted

with the approval of University of the Malaya

Ethics Committee. The research involved a 51-yr-old

female volunteer whose lower limbs were amputated

(bilateral transtibial) because of peripheral vascular

disease. The patient had been classified with

the mobility grade $\mathrm{K} 2$ (the ability to ambulate and

cross environmental obstacles such as stairs, curbs,

or uneven surfaces) according to the American Academy

of Orthotists\&Prosthetists grading system.11 She

had bony residual limbs with adventitious bursa 12 and

no soft tissue or muscle at the distal end (Fig. 2A, B).

She had been using two transtibial prostheses 13 that

contained a silicone liner with pin, shuttle lock, and

Multiflex feet for more than $10 \mathrm{yrs}$. She was referred

to the Brace and Limb Laboratory, University of

Malaya, because of pain at the end of the residual

limbs, especially during the swing phase of gait.

The following components were used to fabricate

four transtibial prostheses (Fig. 1AYD): Dermo

liner and shuttle lock (Icelock-clutch 4 H214 L 

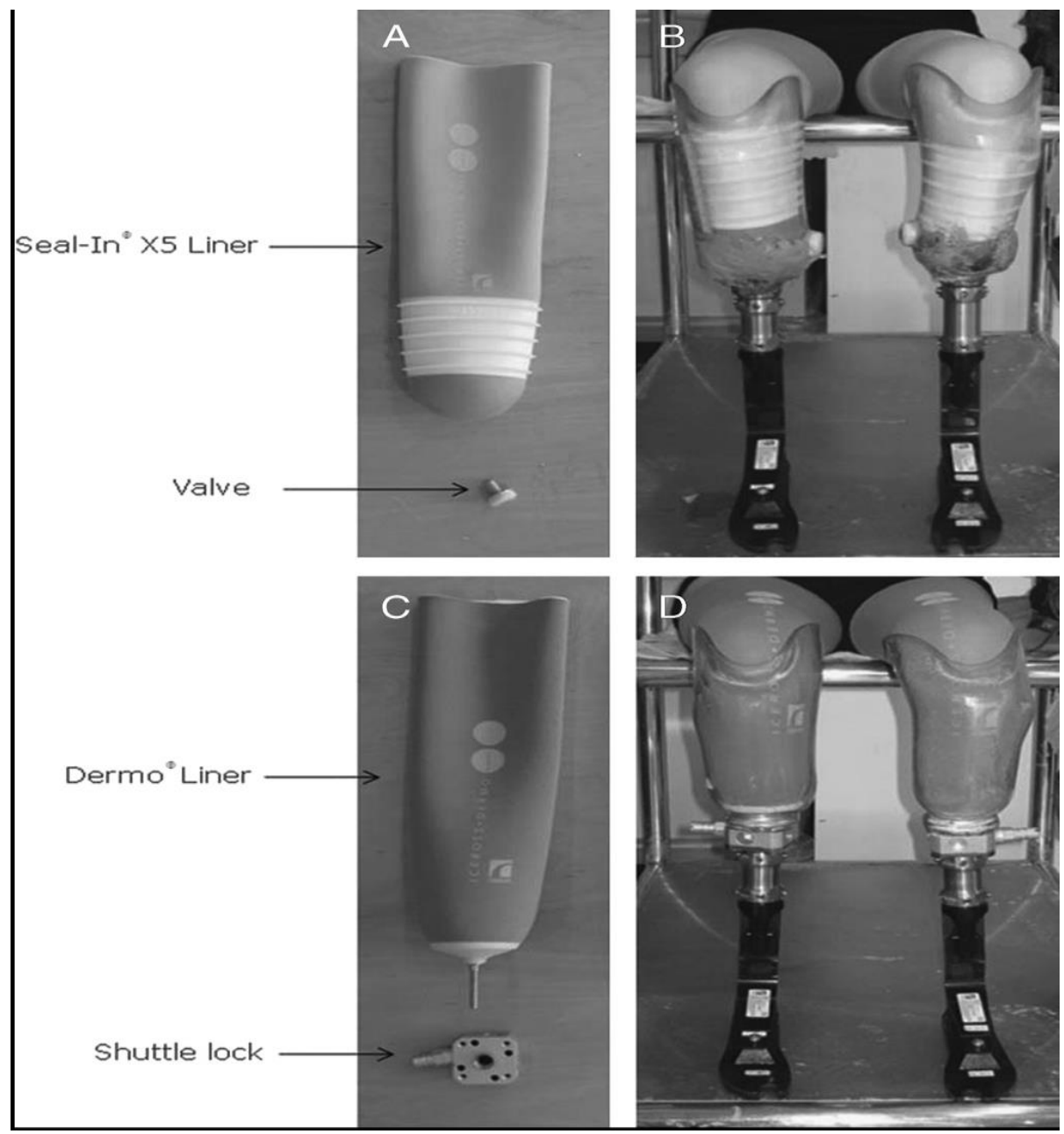

FIGURE 1 The subject wearing the following: Seal-In X5 liner and valve (A and B); Dermo liner and shuttle lock (C and D). 


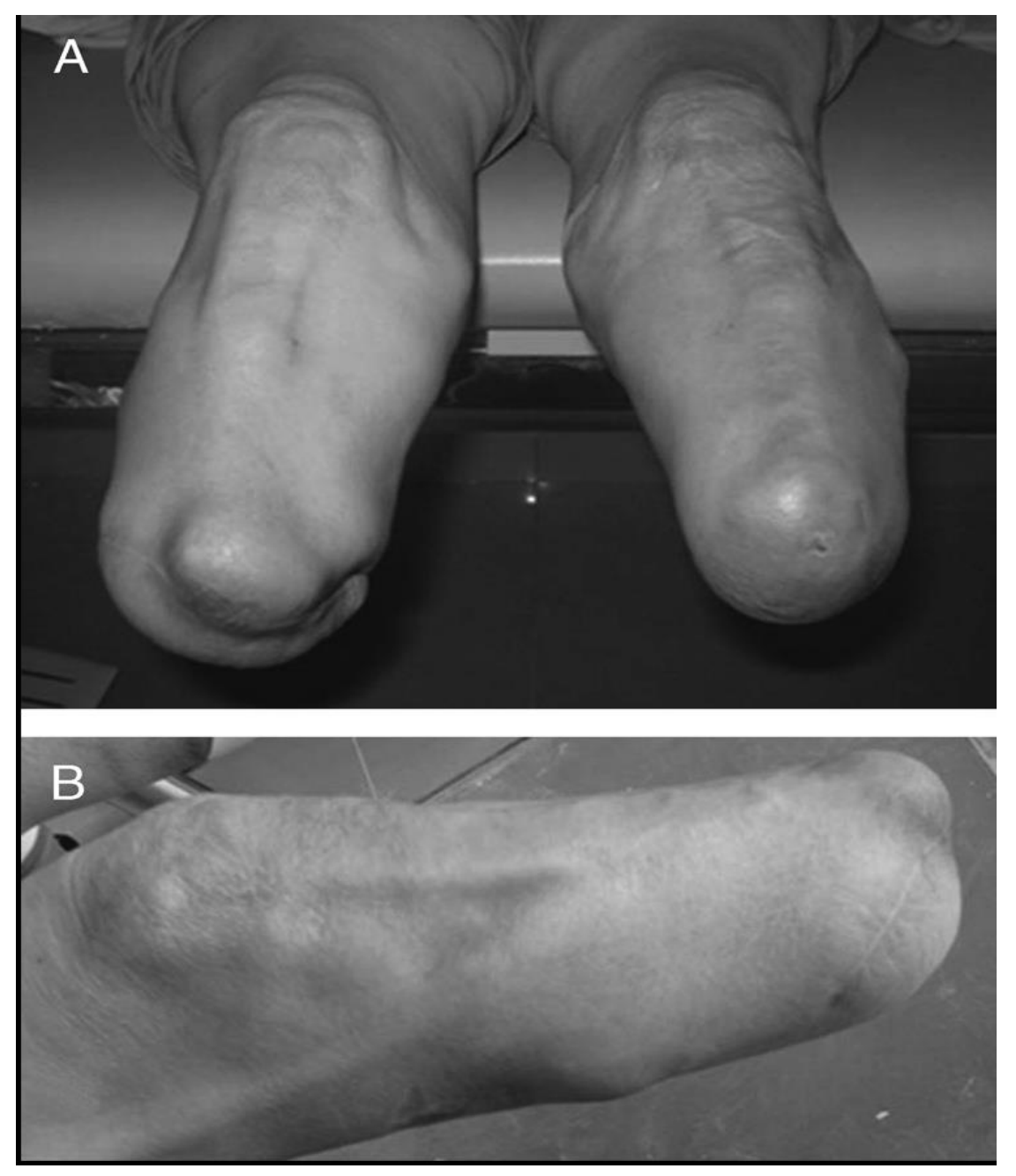

FIGURE 2 The subject's residual limbs in anterior (A)

and lateral views (B). Note the adventitious

bursa over the distal-anterior ends of the residual limbs.

214000), Seal-In X5 liner and valve (Icelock Expulsion Valve 551), double-ended adapters, and Flex-Foot Talux. The prostheses were designed and aligned by one registered prosthetist and orthotist to avoid the variability caused by fabrication, fitting, and alignment technique. The subject was fitted with transparent check sockets to ensure that the sockets were total surface bearing. 14

Once the fitting was confirmed, the patient was asked to use each pair of the new prostheses for 
2 wks to adapt to the new liners and prosthetic feet. After this period, the pistoning inside the socket of each prosthesis was determined by calculating the possible vertical movement between the socket and liner. To identify the pistoning movement inside the prosthetic socket, the following equipments 15 were used: (1) 30-, 60-, and 90-N loads; (2) a camera (Sony A, alpha, DSLR-A200K); (3) two reference rulers attached to the lateral side of the limb and the socket (Fig. 3AYC) to measure the real displacement on the photographs; and (4) markers (two on the socket and two on the liner).

In different static positions, photographs were taken from a fixed distance in such a way that the markers and the reference rulers could be clearly observed. We also made sure that they were not at an angle from the camera stand. The static positions consisted of (1) subject standing with full weight bearing on each prosthetic limb (unilateral stance); (2) subject standing without bearing weight on one prosthesis with the knee extended; and (3) applying the 30-, 60-, and 90-N loads, 16 consecutively, along the longitudinal axis of the prosthesis. The unilateral stance was considered the baseline position, with which all other positions were compared (Fig. 3AYC).

The loads were attached to the prosthetic feet via wire16 to simulate the traction developed at the residual limbYsocket interface during the swing phase of gait.

These conditions were repeated for each of the right and left legs. The subject performed the abovementioned positions three times, and the average values were used for the purpose of the statistical analysis. The accuracy of this measuring system had been previously evaluated by the authors. 15 

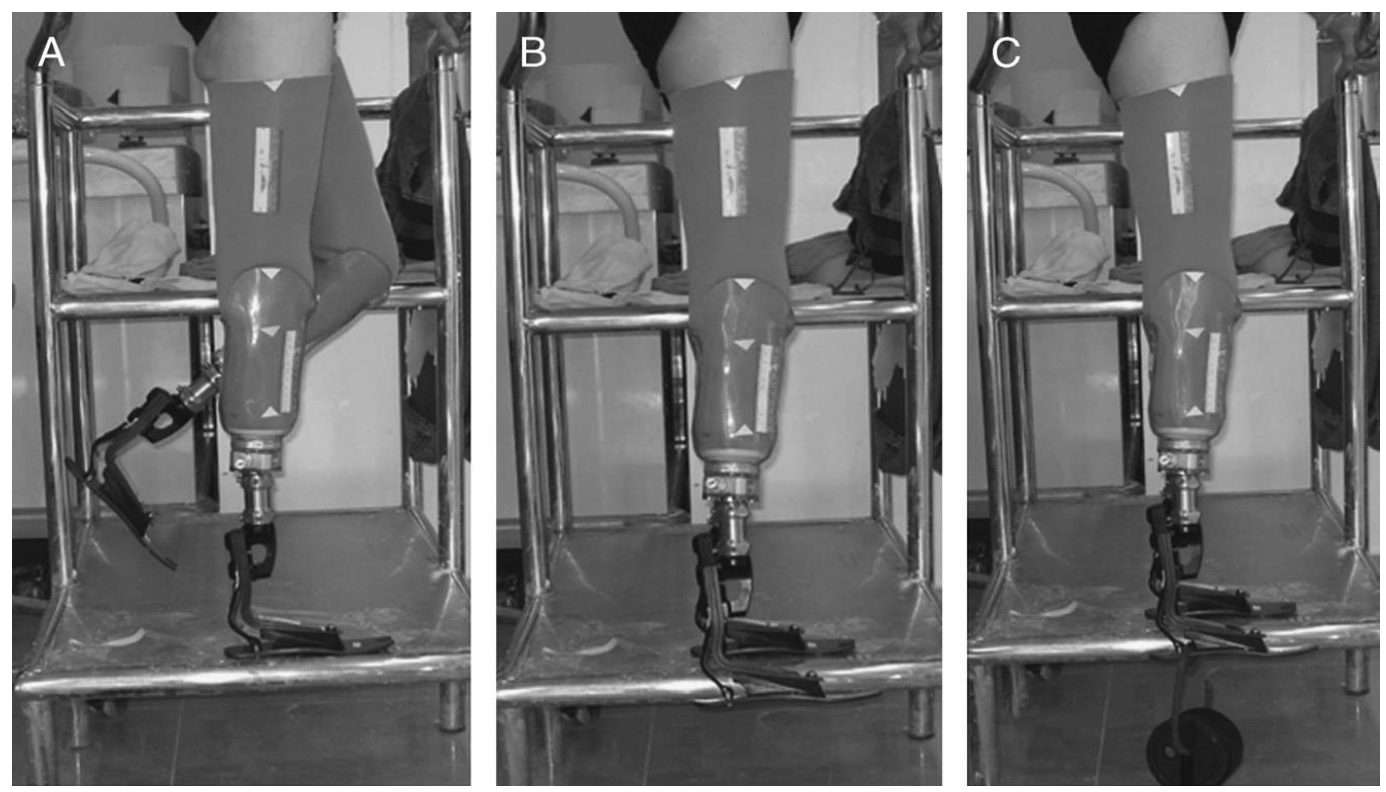

FIGURE 3 The static positions used for this study; full weight bearing (A), non Yweight bearing (B), and adding the loads $(\mathrm{C})$.

Finally, a questionnaire survey was conducted

to obtain the subject's opinion concerning

the liners. The patient was requested to complete two questionnaires for each liner type after 2 wks of continuous prosthetic use. The questionnaires included questions regarding the prosthetic fit, ability to don and doff the prosthesis, ability to walk with the prosthesis, presence of pain in the residual limb, skin traction at the end of the residual limb, and overall satisfaction with each liner. Some elements of the Prosthesis Evaluation Questionnaire (PEQ) questionnaire were used for the purpose of this assessment

The results revealed that the Seal-In X5 liner decreased pistoning inside the socket (Fig. 4) and skin traction and pain at the end of the residual limbs. The subject also found the prostheses to be more comfortable during walking because, according to her, the pressure was distributed uniformly at the distal end of the residual limbs.

\section{Full text is available at :}


http://graphics.tx.ovid.com/ovftpdfs/FPDDNCLBPCEOKH00/fs047/ovft/live/gv024/00002060/00002060-

201210000-00011.pdf

http://www.ncbi.nlm.nih.gov/pubmed/22173083

http://journals.Iww.com/ajpmr/Abstract/2012/10000/Clinical Evaluation of Two Prosthetic Suspension.11.as

px 